\title{
Comprendre la dynamique et les enjeux de coordination des parcours de santé : proposition d'un cadre d'analyse à partir des phénomènes de rupture
}

\section{AUTEURS :}

- Isabelle AUBERT, doctorante, isabelle.aubert@mines-paristech.fr

- Frédéric KLETZ, enseignant-chercheur, frederic.kletz@mines-paristech.fr

- Jean-Claude SARDAS, enseignant-chercheur, jean-claude.sardas@minesparistech.fr.

Centre de Gestion Scientifique, Mines ParisTech, 60 boulevard Saint-Michel 75006 PARIS 


\title{
Comprendre la dynamique et les enjeux de coordination des parcours de santé : proposition d'un cadre d'analyse à partir des phénomènes de rupture
}

\section{Résumé}

La promotion de la logique du parcours, destinée à faire évoluer la prise en charge des patients vers une approche coordonnée et globale, imprègne le champ de la santé depuis une quinzaine d'années en France. Pourtant, l'observation des parcours réels témoigne de la survenue encore fréquente de ruptures dans leur déroulement, entraînée par des désajustements de la réponse aux besoins des patients et des défauts de coordination entre les acteurs. L'objet du présent article est d'analyser les modalités de construction et de déconstruction de cet équilibre précaire et évolutif entre l'offre et les besoins d'aides et de soins, d'en tirer des enseignements sur la notion de rupture, et de proposer un nouveau cadre d'analyse plus adapté à la complexité de cette notion. Nous mobilisons le cadre théorique de la sociologie des parcours de vie (BIDART, 2006, GROSSETTI, 2006, ABBOTT, 2009, BESSIN, 2009), afin d'appréhender les étapes et ingrédients conduisant à une rupture, que nous complétons par le cadre gestionnaire d'analyse de l'activité de coordination des professionnels entre eux et avec le patient, spécifique aux parcours de santé (BLOCH et HENAUT, 2014, FRATTINI et al., 2015). La méthode de recherche adoptée est qualitative et repose sur une étude de cas multiples, fondée sur la reconstitution de parcours de personnes âgées en situation complexe et sur des entretiens réalisés avec les professionnels de santé d'un territoire et une aidante d'un patient. Nos résultats permettent d'enrichir l'analyse des parcours de santé par la mise en lumière du processus et des mécanismes à l'origine des ruptures et, sur ce fondement, de proposer une contribution théorique à la compréhension du phénomène de rupture d'un parcours de santé.

Mots-clé : coordination - parcours - rupture - santé - patients.

\section{Understanding the dynamics and coordination's issues of care pathways : proposition of an analytical framework based on the phenomenon of breakdown.}

\begin{abstract}
The logic of pathway, intended to change the care of patients towards a coordinated and global approach, has been promoted in the health's field for the past fifteen years in France. However, a look on real pathways gives evidence of the still frequent occurrence of breakdowns in their course, caused by disorganizations of the response to the needs of patients, and a lack of coordination between the actors. The purpose of this article is to analyze how this delicate and evolving balance between supply and needs for aids and care is constructing and deconstructing, to draw lessons on the concept of breakdowns, and propose a new framework, which better fits with the complexity of this notion. We rely on both the theoretical framework of the sociology of life pathways (BIDART, 2006, GROSSETTI, 2006, ABBOTT, 2009, BESSIN, 2009), in order to understand the stages and constituents leading to a breakdown, completed by the managerial analysis of the coordination activity of professionals among themselves and with the patient, specific to the health pathways (BLOCH and HENAUT, 2014, FRATTINI and al., 2015). The research method adopted is of
\end{abstract}


a qualitative type and is based on a multiple cases study, grounded on the reconstruction of elderly people's pathways in complex situations and interviews carried out with local health professionals and a patient's caregiver. Our results complete the analysis of health pathways by shedding light on the process and mechanisms leading to breakdowns and, on this basis, allow us to propose a theoretical contribution to the understanding of the phenomenon of breakdown in a health pathway.

Key words : coordination - pathway - breakdown - health - patients. 
L'omniprésence du vocable de «parcours » dans différents champs de la vie économique et sociale a été soulignée par de nombreux auteurs (BOUQUET et DUBECHOT, 2017, JAEGER, 2017, GANNE, 2017). Le secteur de la santé n'échappe pas à cet engouement, qui s'est traduit par l'émergence et la coexistence de deux concepts voisins, le parcours de soins et le parcours de santé, unis par une vision commune de prises en charge coordonnées et globales (BLOCH et HENAUT, 2014). Depuis une quinzaine d'années, les pouvoirs publics encouragent les professionnels à adopter des modes de pensée et d'action relevant de cette logique, pour remédier aux problèmes engendrés par l'approche cloisonnée, renforcée par le raisonnement à l'acte, jusqu'ici dominante (MINVIELLE, 2018) : défauts de coordination entre les professionnels conduisant a minima à des retards dans les prises en charge, une perte de qualité (errances diagnostiques et thérapeutiques, absence d'accès aux soins...), et des dépenses inutiles (hospitalisations inadéquates, répétitions inutiles d'actes...).

Pourtant, on observe encore couramment des situations de parcours accidentés, voire de véritables ruptures, se traduisant par la remise en question brutale de la prise en charge et de la santé du patient. Les mécanismes aboutissant à de telles situations restent aujourd'hui incomplètement explorés par la littérature académique. Les études existantes soit sont centrées sur la consommation de soins, dans une visée typologique des trajectoires des patients (CITTEE et al., 2015, NESTRIGUE et al., 2019), soit tentent d'apprécier les effets d'une organisation respectant la logique du parcours sur la qualité des prises en charge, la satisfaction des patients et des professionnels (VANHAECHT et al., 2009, VANHAECHT et al., 2012, DENECKERE et al., 2012), soit enfin se consacrent à l'analyse des dispositifs de coordination mobilisés au cours des prises en charge (BLOCH et al., 2011 a et b, MATHIEUFRITZ et ESTERLE, 2013). Malgré les apports de ces travaux, notamment sur l'analyse des difficultés de coordination, la caractérisation des ruptures de parcours reste insuffisamment approfondie pour bien appréhender d'une part la nature et les fondements organisationnels de ces ruptures, et d'autre part la manière dont les nouvelles approches orientées parcours devraient permettre d'y remédier.

Dans cet article, nous nous efforçons d'avancer dans la caractérisation des ruptures de parcours, en analysant le parcours comme un processus de formation d'un équilibre évolutif et précaire entre les besoins du patient et l'offre de soins et d'aides. Notre analyse est centrée sur des parcours complexes de personnes âgées en perte d'autonomie, plus particulièrement exposées au risque de rupture. Dans son rapport «Assurance Maladie et perte d'autonomie » du 23 juin 2011, le Haut Conseil pour l'Avenir de l'Assurance Maladie pointe ainsi une dégradation de la qualité du «trajet de santé » des personnes avec l'âge, corrélée à des difficultés d'articulation entre les dispositifs visant à prendre en charge la perte d'autonomie et les dispositifs centrés sur le soin. Cette fragmentation se conjugue avec des inégalités d'accès aux soins, des difficultés de financement, et une insuffisance des capacités et des prestations en institution gériatrique (SAINT-JEAN, 2008).

Notre approche théorique s'inscrit dans un double cadre théorique : d'une part, celui proposé par la sociologie sur les parcours de vie, qui explore la survenue de «bifurcations biographiques » (BESSIN, 2009, BESSIN et al., 2009, BIDART, 2006) ou de «turning points » (ABBOTT, 2009) dans ces parcours, potentiellement assimilables aux ruptures observables dans les parcours de santé, en raison de l'imprévisibilité du moment précis de leur survenue et de leur issue, ainsi que d'une forte irréversibilité potentielle des conséquences (GROSSETTI, 2006); d'autre part, le cadre gestionnaire de l'analyse de l'activité de coordination, spécifique aux parcours de santé, qui relie les professionnels entre eux et avec le patient (BLOCH et al., 2011a, BLOCH et HENAUT, 2014, FRATTINI et al., 2015). 
Notre recherche repose sur une méthode qualitative. Dans le cadre d'une demande d'accompagnement formulée par une Agence Régionale de Santé (ARS) sur la mise en place de cette nouvelle logique de parcours, nous avons procédé à la reconstitution chronologique de dix parcours de personnes prises en charge par un dispositif d'appui à la coordination $\left(\right.$ CLIC $^{1}$, MAÏA $^{2}$ ) ou un EHPAD ${ }^{3}$. Cette approche processuelle (LANGLEY et al., 2013) permet de mettre en lumière l'enchaînement temporel des événements jalonnant les parcours, et de comprendre les processus dynamiques qui les structurent, mais aussi l'occurrence de ruptures dans ce processus. Nous avons croisé cette analyse avec la vision des parcours développée par les professionnels du territoire (médecins généralistes, représentants d'établissements hospitaliers, responsables des dispositifs d'appui à la coordination, équipe de management d'EHPAD) rencontrés lors de 15 entretiens semi-directifs, et par une aidante d'un patient dont nous présentons la situation dans l'article.

Nos résultats permettent d'enrichir l'analyse des parcours de santé par la mise en lumière du processus et des mécanismes à l'origine des ruptures et, sur ce fondement, de proposer une contribution théorique à la compréhension du phénomène de rupture d'un parcours de santé

Après avoir resitué l'émergence de la logique du parcours au regard des enjeux actuels du système de santé, nous détaillons les orientations théoriques qui structurent notre réflexion, puis notre méthodologie, avant de présenter et de discuter nos résultats.

\section{Brève généalogie et caractérisation de la logique du parcours}

Face aux défis soulevés par le vieillissement de la population et le développement des maladies chroniques, deux exigences se sont imposées aux acteurs du système de santé : celle d'une coordination et d'une coopération accrues, afin d'assurer la continuité et la cohérence des prises en charge (BLOCH et al., 2011), et celle d'une évolution de l'offre de soins et de services, dans une logique de prise en charge globale et s'adaptant à des besoins multiples et évolutifs. La notion de parcours a émergé dans l'espace public comme une reformulation de ces impératifs, sous différentes appellations : parcours de soins, parcours de santé et parcours de vie. Elle traduit le passage d'une logique de prise en charge fondée sur la juxtaposition d'actes indépendants à un regard plus transversal, prenant en compte l'interdépendance des actes et des intervenants.

L'approche du «parcours de soins » a été historiquement développée à partir des années 1980 aux Etats-Unis, puis s'est diffusée dans les pays occidentaux. Ses promoteurs le présentent comme une modélisation du processus de soin, élaborée sur la base de recommandations de bonnes pratiques (LAWAL et al., 2016, KINSMAN et al., 2010). Raisonner en termes de parcours apparaît ainsi comme une démarche de rationalisation de la planification et de l'articulation des pratiques médicales et soignantes, réunissant les intervenants dans une approche partagée des finalités et du déroulement de la prise en charge. Cette approche se prête bien à des parcours de niveau de complexité modéré, relativement prévisibles dans leur déroulement, mais paraît peu adaptée à des parcours complexes, dont le déroulement peut difficilement être prédéfini.

Selon BLOCH et HENAUT (2014), la prise en compte de la complexité de certains parcours a conduit à la promotion depuis le début des années 2010, d'approches relevant des «parcours de santé » et des «parcours de vie », qui appréhendent de manière globale et personnalisée

\footnotetext{
${ }^{1}$ Centre Local d'Information et de Coordination

${ }^{2}$ Méthode d'Action pour l'Intégration des services d'aides et de soins dans le champ de l'Autonomie

${ }^{3}$ Etablissement d'Hébergement pour Personnes Agées Dépendantes
} 
l'accompagnement des personnes, en intégrant des dimensions de prévention, d'éducation thérapeutique et de prise en charge sociale et médico-sociale (parcours de santé), ainsi que l'ensemble des déterminants - logement, éducation, emploi... - influençant leur vie parcours de vie). De nouvelles compétences s'avèrent dès lors nécessaires pour prendre en charge les parcours, allant de la coordination à l'élaboration et la mise en œuvre de réponses aux besoins multidimensionnels des personnes.

\section{Comprendre la dynamique des parcours réels : synthèse des approches développées dans la littérature}

Nous rappelons tout d'abord les enseignements de la littérature sur les parcours de soins et de santé, puis nous explorons le potentiel heuristique de l'approche sociologique des parcours de vie.

\subsection{Apports et limites des études typologiques, d'impact et organisationnelles}

La littérature sur les parcours peut être classée en trois catégories: les approches typologiques, les études d'impact et les approches organisationnelles.

Les approches typologiques, centrées généralement sur une pathologie, détaillent l'itinéraire et la succession des étapes dans la prise en charge (lieux et intervenants) de la pathologie sur un temps et un territoire donnés. Les analyses de CITTEE et al. (2015) sur la BronchoPneumopathie Chronique Obstructive (BPCO) et de NESTRIGUE et al. (2019) sur les Accidents Vasculaires Cérébraux (AVC) illustrent cet angle d'analyse. Comme c'est le cas dans le deuxième article cité, ces études peuvent être prolongées par une analyse des déterminants des trajectoires, fondée sur des corrélations statistiques entre des variables caractéristiques des parcours.

Les études d'impact interrogent les effets d'une organisation orientée selon une logique du parcours (lui-même fondé sur des recommandations de bonnes pratiques) sur la qualité des prises en charge des patients et le travail des professionnels, à partir d'essais contrôlés randomisés (VANHAECHT et al., 2012) ou de questionnaires administrés aux professionnels ou aux patients (DENECKERE et al., 2012, VANHAECHT et al., 2009). Ces études concluent à un effet positif sur la qualité globale des prises en charge et sur deux de leurs composantes : la coordination des acteurs et la continuité du processus. Certaines études qualitatives visent également à mesurer et comprendre les écarts entre les recommandations de bonnes pratiques et les «parcours constatés » (GACHE et al., 2014).

Enfin, les approches organisationnelles s'intéressent au rôle et aux éventuelles limites des dispositifs, au sens des acteurs et des outils, assurant l'identification des besoins des personnes et leur couverture, ainsi que la coordination au sein des parcours. Ainsi, GAND et al. (2014) proposent une modélisation des besoins de la dyade aidé/aidant, selon une approche multidimensionnelle et dynamique permettant de comprendre comment l'aidant parvient ou non à assumer son rôle, et mettent en regard les services existant pour répondre à ces besoins. Sur un plan micro, BLOCH et al. (2011b) analysent le positionnement des gestionnaires de cas d'une MAÏA par rapport à d'autres acteurs de la coordination et à un réseau d'intervenants déjà implanté. MATHIEU-FRITZ et ESTERLE (2013) étudient de leur côté l'échec, en tant qu'outil de coordination, du dossier patient informatisé déployé en Picardie.

$\mathrm{Au}$ total, ces différentes études mettent en évidence les enjeux organisationnels liés au décloisonnement des secteurs et à l'amélioration de la coordination entre acteurs, et les enjeux relatifs à la survenue de transitions biographiques ou de ruptures identitaires (HENAUT et 
SARDAS, 2012).Elles ne rendent toutefois pas toujours compte explicitement de la dynamique des parcours, et plus précisément de la manière dont se constituent les équilibres et déséquilibres - entre les besoins des patients et les services offerts par le système de soins et d'aides.

\subsection{L'éclairage de l'approche sociologique des parcours de vie}

Afin d'éclairer cet aspect peu exploré, nous proposons de mobiliser l'analyse sociologique des parcours de vie, en reprenant plusieurs de ses dimensions explicatives.

La première réside dans l'adoption d'une approche longitudinale, à l'image des analyses relevant du courant des « process studies » (LANGLEY et al., 2013) car le temps constitue un facteur d'intelligibilité des parcours à prendre en compte comme tel. Dans la sociologie des parcours de vie, il est saisi selon trois échelles. La première, biographique, permet de comprendre les spécificités individuelles d'un parcours, la deuxième, sociale, renvoie aux influences familiales et sociales du groupe auquel l'individu appartient, et la troisième, historique, fait référence aux déterminants sociétaux et historiques des parcours. L'interaction entre ces dimensions, déclinées selon des caractéristiques objectives et les perceptions subjectives des individus (BIDART, 2006) rend compte de la spécificité de chaque parcours. Un dernier apport heuristique de la sociologie des parcours de vie concerne l'étude des ruptures de parcours, parfois nommées «bifurcations » ou «turning point ». Les bifurcations sont entendues comme "l'apparition d'une crise ouvrant un carrefour biographique imprévisible, dont les voies sont elles aussi au départ imprévues (...). [et] au sein desquelles sera choisie une issue qui induit un changement important d'orientation »(BIDART, 2006). Cette définition est proche de celle de «turning point » d'ABBOTT (2009), qui le présente comme un moment où l'individu sort d'une trajectoire pour en emprunter une nouvelle, du fait d'une dissolution de ses réseaux de relations habituels.

A partir d'une enquête longitudinale menée auprès de jeunes entrant dans la vie adulte, BIDART (2006) détaille les étapes de ces ruptures, conduisant à la déstructuration de la cohérence initiale entre la situation d'un jeune dans différents domaines (personnel, familial, professionnel) et ses valeurs et capacités, puis à la construction d'une nouvelle cohérence dans la situation postérieure à la bifurcation. Elle montre que le processus par lequel la bifurcation advient, intègre une «période critique » préalable, durant laquelle la situation se dégrade, et qui se clôt par un moment-clé marqué par un ou plusieurs événements. La bifurcation survient lorsqu'un événement se produisant dans l'une des sphères de la vie, produit des effets dans d'autres sphères, entraînant une remise en question globale de la logique de la trajectoire jusqu'alors suivie.

Son analyse révèle la diversité des ingrédients objectifs et subjectifs, interagissant entre eux, qui participent à la recomposition des trajectoires. Les éléments objectifs rassemblent des déterminants macro-sociaux, par exemple le système de droits et d'aides auquel la personne a accès, des ingrédients à un niveau méso-social tels que le territoire où elle vit et le soutien familial dont elle bénéficie, et enfin, à l'échelle individuelle, ses caractéristiques personnelles et son expérience de vie. Les éléments subjectifs, également situés aux trois échelles citées, alimentent l'éclairage donné par la personne sur le poids des différents atouts et contraintes qui ont influencé ses décisions.

Dans la suite de l'article, nous verrons comment cette approche, complétée par une analyse organisationnelle de l'activité de coordination, peut nous permettre de mieux comprendre la survenue de ruptures ou leur évitement. 


\section{Méthodologie}

Notre recherche se fonde sur une méthodologie qualitative, développée à travers une étude de cas multiples sur un territoire.

\subsection{Présentation du territoire investigué}

Le territoire sur lequel nous avons collecté nos données recouvre deux arrondissements parisiens. La population y est vieillissante ${ }^{4}$ et présente une prévalence d'ALD (Affection de Longue Durée) supérieure à la moyenne francilienne pour certaines pathologies chroniques, comme les AVC, les pathologies cancéreuses, le VIH, les troubles de la personnalité et les maladies coronariennes, nécessitant un suivi au long cours et une coordination entre de nombreux acteurs ${ }^{5}$. L'offre de soins, médico-sociale et d'accompagnement social est importante et diversifiée. A titre d'exemple, la densité de l'offre de soins libérale y est supérieure à la moyenne de l'Ile-de-France, quel que soit le type de professionnel étudié : médecin généraliste, spécialiste, infirmier, kinésithérapeute.... Dix établissements de santé, publics ou privés, maillent le territoire. Au regard de ces caractéristiques, les enjeux autour de l'organisation des parcours semblent renvoyer à l'articulation de ces nombreux acteurs, ainsi qu'à l'organisation du maintien à domicile de personnes âgées, polypathologiques et dépendantes.

Dans ce territoire, des professionnels ont souhaité s'investir dans l'amélioration de la coordination des parcours, en s'engageant dans la création de deux Communautés Professionnelles Territoriales de Santé (CPTS), le déploiement d'un projet d'Initiative de Prise en charge Partagée (IPEP) au titre des expérimentations autorisées par l'article 51 de la Loi de Financement de la Sécurité Sociale 2018, relatif à 1'amélioration des parcours des personnes âgées de plus de 75 ans, et enfin la participation au programme national e-parcours, destiné à aider des acteurs prêts à s'engager dans des actions collectives d'amélioration des parcours, par la mise à disposition d'outils numériques de partage d'informations.

\subsection{Collecte et analyse des données}

Les données auxquelles nous avons eu accès proviennent de différentes structures du territoire (CLIC, MAÏA, EHPAD), qui nous ont transmis des documents relatifs à des prises en charge de situations complexes: fiches du Formulaire d'Analyse Multi-dimensionnelle et d'Orientation (FAMO), échanges de mails avec les différents partenaires sur la situation, rapports d'évaluation effectuées au domicile des patients, synthèses, comptes-rendus de réunions, et plans personnalisés de soins. Les situations devaient rendre compte de parcours présentant des ruptures avérées ou évitées. Au total, nous avons collecté les données de dix parcours, trois traités par le CLIC, quatre par la MAÏA, un conjointement par le CLIC et la MAÏA, et deux par l'EHPAD. Les parcours ont été reconstitués selon une logique chronologique, puis analysés, en mettant en exergue les principales étapes de leur déroulement, le rôle des différents acteurs, ainsi que les enjeux de coordination soulevés par chacun d'eux. Cette étude a été autorisée par la CNIL.

Nous avons croisé ces données avec celles obtenues lors de quinze entretiens semi-directifs réalisés avec les membres du comité de projet e-parcours, réalisés entre novembre 2018 et juin 2019 : médecins généralistes (3), représentants de directions hospitalières (4),

\footnotetext{
${ }^{4}$ La part des personnes âgées de 75 ans et plus est passée de $7.7 \%$ de la population en 2011 à $8 \%$ en 2016 dans l'un des arrondissements et de $8.3 \%$ à $8.7 \%$ sur la même période dans l'autre arrondissement (données INSEE).

${ }^{5}$ Source : dossier d'analyse du projet de participation au programme e-parcours - ARS
} 
représentants de deux M2A $\mathrm{A}^{6}$ (4), directrice d'EHPAD (1), chefs de projet e-parcours (2), représentants de la direction départementale de l'ARS (1). Nous avons évoqué avec eux leur vision des parcours sur le territoire, leurs objectifs et leurs attentes par rapport au projet eparcours, et les questions de gouvernance territoriale autour de l'analyse et du traitement des problématiques de parcours. Nous avons également pu nous entretenir avec l'épouse d'un des patients (M. C.), dont nous retraçons le parcours dans la suite de l'article.

Les données collectées lors des entretiens ont été analysées par grand type d'acteurs, en montrant les points communs et les nuances existant dans leur approche des parcours, et dans leur analyse des besoins de coordination entre professionnels et entre organisations.

En décembre 2019 et février 2020, nous avons restitué devant le comité de projet l'ensemble de nos analyses, et partagé des pistes de réflexion sur les moyens d'améliorer le déroulement des parcours.

\section{Résultats : Les parcours complexes et leurs ruptures : à la recherche d'un cadre d'action global et partagé}

La partie qui suit présente plusieurs parcours de personnes âgées, qui mêlent des problématiques sociales et médicales, à l'origine de la complexité des situations et de la difficulté à trouver un équilibre entre offre et besoins de soins et d'aides. Ces parcours se présentent comme une somme de micro-choix visant à atteindre un compromis, toujours instable et remis en question, entre l'évaluation des besoins, les ressources disponibles sur le territoire, et les différentes représentations des acteurs concernés. Ils mettent en lumière les configurations de relations et d'interactions qui structurent la prise en charge (ou les tentatives de prise en charge) des personnes.

\subsection{Des configurations à risque : la conjonction de l'aggravation de la dépendance avec la défaillance de l'aidant et le refus d'aide.}

Nous illustrons cette configuration avec l'histoire de Madame A., âgée de plus de 80 ans et décédée en 2019, dans une situation de rupture avérée. Elle a été prise en charge par la MAÏA de juin 2018 à mars 2019. Elle était célibataire et sans enfant.

Rappelons tout d'abord que la MAIA est une démarche qui associe les acteurs engagés dans l'accompagnement des personnes âgées en perte d'autonomie et de leurs aidants et vise à éviter les ruptures de prise en charge. Pour soutenir cette démarche, des "gestionnaires de cas » sont dédiés au traitement des situations complexes, comme celle de Mme A.

Sa situation a été signalée par la directrice du service d'aide à domicile (SAAD) qui intervenait chez elle. Le FAMO mentionne un refus d'aide de la part de Mme A. (malgré l'existence d'un plan d'aides), à l'origine d'une hygiène personnelle dégradée, et un isolement social important : Mme A. ne sort jamais et a pour unique contact sa sœur qui vient la voir le dimanche. Mme A. entretient une relation difficile avec les intervenants à domicile, conduisant à leur épuisement et à des changements fréquents. La gestionnaire de cas désignée pour suivre Mme A. propose de la rencontrer, mais celle-ci refuse, en précisant ne pas comprendre l'intérêt de son intervention. Sa sœur tient un discours identique. La gestionnaire parvient à suivre ponctuellement la situation par l'intermédiaire de la directrice du SAAD. A l'automne 2018, celle-ci l'informe d'une altération de l'état de santé de Mme A., qu'elle a

\footnotetext{
${ }^{6}$ Maison des Aînés et des Aidants, regroupant CLIC, MAÏA et réseau.
} 
trouvée pâle et amaigrie lors de sa dernière visite. Fin 2018, le Docteur X accepte de devenir médecin traitant de Mme A., et la gestionnaire demande à la directrice du SAAD de la tenir informée de l'état de santé de Mme A., pour le faire intervenir si besoin. En février 2019, la gestionnaire tente à nouveau de rencontrer Mme A., mais celle-ci ne répond pas. En mars 2019, la gardienne de l'immeuble l'appelle pour l'informer de la récente hospitalisation de Mme A. La gestionnaire contacte sa sœur, qui lui apprend le décès de Mme A., survenu à l'hôpital dans lequel elle avait été récemment admise à deux reprises.

Au total, la situation de Mme A. est restée globalement insaisissable pour le système d'aides et de soins. Isolement, aggravation de la dépendance et refus d'aides et de soins se sont conjugués pour créer les conditions d'une rupture, difficile à éviter au regard du faible espace d'action laissé par la patiente aux intervenants. Ce cas illustre une facette des problématiques de la coordination, en montrant d'une part l'importance de cette fonction de coordination face à des professionnels ayant un domaine d'expertise circonscrit, et d'autre part l'ampleur des difficultés pour l'assurer dans un contexte de refus de soins et d'aides.

\subsection{Des problématiques de coordination entre les professionnels qui freinent l'élaboration d'une vision partagée}

Le parcours de Mme B. témoigne d'une autre dimension des problématiques de coordination, et s'inscrit dans une situation déficitaire au regard de ses besoins. Âgée de plus de 80 ans, veuve avec une fille, Mme B. est prise en charge à partir de juin 2018 par différents services, service social polyvalent (SSP), réseau de soins et CLIC. Ce dernier est un lieu d'accueil de proximité destiné à fournir aux personnes âgées et à leurs familles information, conseil et orientation. Dans chaque CLIC, des coordonnateurs sont chargés d'organiser l'action des différents intervenants médicaux et sociaux, les cas les plus complexes étant toutefois transmis aux MAIA.

En réponse à la saisine d'un hôpital, et après un refus initial de Mme B., le réseau procède à l'évaluation de ses troubles cognitifs en septembre 2018. Le compte-rendu ne sera transmis au CLIC que plusieurs mois plus tard (janvier 2019), en dépit de multiples relances. Pendant cette période, les pompiers se déplacent très fréquemment au domicile de Mme B., pour des chutes à répétition, parfois suivies de brefs passages à l'hôpital. En octobre, Mme B. est à nouveau hospitalisée en urgence pour une décompensation cardiaque. Sa fille souhaite qu'une mesure de mise sous protection juridique soit prise, mais la patiente, rencontrée par une assistante sociale du SSP, refuse. A cette époque, le CLIC et le SSP mènent des démarches parallèles, sans que ni l'un, ni l'autre ne se reconnaissent comme officiellement coordonnateur de la situation.

Le $1^{\text {er }}$ mars 2019, une infirmière coordinatrice du réseau provoque une réunion de synthèse entre tous les intervenants, et avec la fille de Mme B. La réunion aboutit à une proposition d'un plan d'aides global, et acte la préparation d'un dossier pour une entrée en EHPAD, par souci d'anticipation. Dans les semaines suivantes, Mme B. est à nouveau hospitalisée à plusieurs reprises, à la suite de chutes. En avril 2019, date à laquelle nous avons réalisé notre collecte de données, la situation était inscrite sur la liste d'attente de la gestion de cas.

Outre la complexité intrinsèque de ce parcours chaotique, la reconstitution chronologique des événements montre les effets négatifs de l'absence de désignation d'un référent de la situation, ainsi que du manque de dialogue entre les professionnels et de partage sur les objectifs de la prise en charge (jusqu'en mars 2019). Ce cas illustre ainsi la persistance de 
cloisonnements entre les secteurs et les difficultés de coordination, y compris entre les professionnels dont c'est le métier.

\subsection{Des accompagnements personnalisés et globaux, favorisant le maintien sécurisé à domicile}

A l'inverse, d'autres prises en charge démontrent la possibilité d'éviter des ruptures et de créer un accompagnement adapté, au long cours, de situations de dépendance parfois très lourde au domicile, comme l'illustre le parcours de M. C.

M. C., âgé de plus de 85 ans, vit à son domicile, avec son épouse. Il est atteint de démence et souffre de différents problèmes somatiques (diabète, hypertension artérielle...). En 2015, il intègre l'accueil de jour d'un EHPAD du territoire, où il se rend deux fois par semaine. $\mathrm{Au}$ départ mal acceptée par le couple, cette prise en charge se révèle satisfaisante avec le temps pour M. C. et pour Mme C., à qui elle offre un répit. En 2016, M. C. réalise un premier séjour temporaire à l'EHPAD, afin de permettre à son épouse de se reposer. Ses troubles cognitifs et sa dépendance s'aggravent, nécessitant une aide totale dans tous les actes de la vie quotidienne. Il se déplace désormais en fauteuil roulant. M. C. effectue entre 2017 et 2019 plusieurs séjours temporaires à l'EHPAD, qu'il accepte bien. Sa prise en charge au domicile est par ailleurs très dense, avec trois passages infirmiers par jour, deux séances hebdomadaires d'orthophonie, et une personne de compagnie (trouvée par l'EHPAD) deux fois par semaine. Dans le cadre de l'accueil de jour, M. C. réalise deux séances de kinésithérapie par semaine. Son épouse bénéfice d'une aide au ménage.

Mme C., qui se dit très soutenue par ses enfants, la psychologue de l'accueil de jour, et par l'équipe de l'EHPAD, a suivi le parcours «Aidant en EHPAD », et participé aux groupes de parole de France Alzheimer. Elle souligne néanmoins qu'elle est très fatiguée, et qu'elle sait qu'elle doit envisager le placement de son mari à l'EHPAD (à temps complet), mais cette perspective reste difficile à accepter.

Dans cette situation, la densité de la prise en charge de Monsieur C. au domicile, couplée à l'orientation vers l'accueil de jour et de manière progressive vers l'hébergement temporaire en EHPAD, témoigne d'une adaptation à ses besoins croissants d'accompagnement, mais aussi de la préoccupation des professionnels de préserver son épouse, fragilisée par l'âge et la confrontation avec la détérioration physique et psychique de son époux. L'approche est donc globale et prend en compte les besoins de l'aidé et de l'aidant. Ce cas met également en relief l'éventail des compétences qu'il faut mobiliser pour accompagner les parcours complexes, afin de s'adapter à des besoins multi-dimensionnels et évolutifs.

\section{Discussion}

Nous développons dans ce chapitre deux enseignements tirés de notre analyse: l'interprétation, sociologique et organisationnelle, des ruptures de parcours et l'approfondissement de la notion de rupture.

5.1. L'interprétation des ruptures de parcours, comme un processus de déconstruction de la cohérence entre les besoins des personnes et l'offre d'aides et de soins 
Notre étude des parcours montre tout d'abord l'apport du cadre d'analyse de la sociologie des parcours de vie pour mieux saisir le phénomène des ruptures de parcours de santé.

En premier lieu, comme lors des bifurcations biographiques, les ruptures des parcours de santé obéissent à un schéma temporel que l'on peut décomposer en plusieurs phases : une « période critique » d'accumulation des difficultés conduisant à une perte de cohérence entre la situation de la personne et les modalités de sa prise en charge, puis la survenue d'un événement souvent exogène, précipitant la «crise », et enfin le choix d'une nouvelle trajectoire, caractérisée par un nouvel équilibre provisoire.

Ainsi, dans le parcours de Mme A., la rupture a été précédée d'une période de dégradation progressive de son état de santé partiellement invisible et sans qu'une prise en charge médicale puisse être instaurée. Mme B. connaît une situation instable, marquée par des recours itératifs aux urgences et des hospitalisations, en l'absence d'un dispositif d'accompagnement complet. Ces situations confirment que la rupture est un processus non nécessairement réductible à l'événement qui provoque un basculement de situation. Celui-ci peut être le révélateur - et parfois le catalyseur - d'un déséquilibre installé progressivement plutôt que sa cause. A contrario, une rupture concernant M. C. a pu être évitée, car sa situation fragile, couplée à celle de sa femme, avait été identifiée et prise en charge précocement.

La période de fragilisation se clôt par un événement, qui précipite la rupture, comme l'illustre l'hospitalisation en urgence de Mme A. Selon la nature et la gravité de la rupture, soit une nouvelle séquence s'ouvre pour les personnes, soit cette rupture met fin définitivement au parcours, comme pour Mme A..

Comme les parcours de vie, les parcours de santé peuvent être analysés à partir de l'intrication entre plusieurs déterminants, objectifs et subjectifs, se situant à différentes échelles sociales et temporelles. Tous les parcours étudiés s'inscrivent dans un contexte sociétal et de politique publique caractérisé par la priorité donnée au maintien à domicile des personnes âgées. Le contexte local met en lumière l'existence de ressources conséquentes, permettant que de nombreux soins et services soient proposés au domicile et en EHPAD. Des ingrédients individuels interfèrent également dans les parcours, comme le niveau de ressources financières des personnes. A ces facteurs objectifs, se mêlent des éléments subjectifs, tels que les contextes familiaux (la sœur de Mme A. conforte par exemple celle-ci dans son refus d'être aidée) et sur un plan individuel, la réticence envers la mise en place d'aides, ainsi que le déni de l'aggravation de la situation.

Au total, ce cadre d'analyse rend compte du caractère processuel et multi-dimensionnel des ruptures de parcours, mais il doit être complété par un regard plus organisationnel portant sur l'analyse de la coordination entre les acteurs.

Toutes les situations étudiées relèvent du type de coordination le plus intense et complexe décrit par CONTANDRIOPOULOS et al. (2001), qui est la «coordination collective», requise quand «les problèmes de santé sont multiples ou complexes, aux contours flous, dont l'évolution dans le temps et dans l'espace est incertaine », et qu'il existe une interdépendance forte des acteurs. Elle repose sur une prise en charge organisée autour du patient et des ententes formelles et informelles entre les intervenants. Ce sont cette organisation et cette entente qui semblent avoir fait défaut dans la situation de Mme B. En leur absence, la qualité et la cohérence de la réponse aux besoins sont affaiblies, contribuant indirectement à l'instabilité de la situation. 
La coopération entre les professionnels doit s'articuler avec la coordination qui les relie aux patients (et à leurs aidants). L'exemple de M. et Mme C. souligne qu'il s'agit d'une condition importante pour parvenir non seulement à éviter une rupture, mais aussi à instaurer une stabilité pérenne de la situation. Ce constat rejoint les analyses sur le rôle des aidants auprès de personnes âgées de GAND et al. (2014), ainsi que les conclusions de BUTHION et GODE (2014), selon lesquelles l'efficacité globale du processus de coordination dépend de la capacité à articuler les pratiques - complémentaires - des coordonnateurs informels que sont les aidants et celles des professionnels. Dans le cas de M. C., l'étude du dossier montre des interactions régulières entre Mme C. et l'équipe de l'EHPAD, par exemple sur le vécu de son époux à l'accueil de jour ou sur son comportement au domicile. Réciproquement, l'équipe accompagne Mme C. dans l'acceptation des placements temporaires de son époux et l'oriente vers des solutions d'aide aux aidants.

\subsection{L'approfondissement théorique de la dynamique des ruptures de parcours}

Notre analyse des parcours nous amène à distinguer trois situations au regard du risque de rupture : les ruptures avérées (qui ont été observées), les situations déficitaires et enfin les ruptures évitées.

En écho aux parties précédentes, nous précisons tout d'abord la nature des évènements que nous considérons comme des ruptures de parcours. Celles-ci recouvrent en premier lieu des situations pour lesquelles l'absence ou le refus (durable) d'une prise en charge entraîne des conséquences délétères pour le patient, compte tenu de son état de fragilité. Elles concernent également les prises en charge en urgence, alors que la situation pouvait être anticipée. Divers évènements illustrent ces deux types de ruptures: des décès précipités, une institutionnalisation sans préparation, une hospitalisation qui aurait pu être évitée (CARTIER et al., 2014), des passages aux urgences répétés, ... Dans ce cas, le désajustement apparaît lorsque se creuse un écart entre la situation (évolutive) de la personne et le mode de représentation de cette situation par les professionnels, ou leur capacité à activer les ressources devenues nécessaires.

Les situations étudiées montrent qu'en plus des ruptures évitées (M. et Mme C.) ou avérées (Mme A.), existe un troisième cas de figure, des situations déficitaires, caractérisées par un désajustement durable entre les besoins et la prise en charge, mais sans remise en question pour l'instant de la trajectoire de vie de la personne : il concerne des situations très précaires au domicile, avec un risque de décompensation brutale, du fait de difficultés tant dans l'adaptation entre les besoins et l'offre de soins et d'aides, que dans la coordination entre les professionnels et avec le patient. Le parcours de Mme B. s'inscrit dans cette catégorie : sa situation est identifiée par les professionnels, mais du fait de la difficulté à prendre en compte ses besoins et d'une coordination mal assurée, des hospitalisations répétées, menacent son maintien à domicile.

Nous complétons cette typologie par une analyse du processus menant à la réalisation ou à l'évitement d'une rupture, ou à l'installation d'une situation de rupture latente. Deux paramètres permettent de différencier les situations : d'une part, la durée du désajustement entre les besoins et leur prise en charge, et d'autre part, l'irréversibilité des conséquences de ce désajustement, déjà identifiée dans les analyses des parcours de vie.

La durée du désajustement qui correspond à la période critique décrite plus haut, est un facteur important de compréhension de certaines ruptures, car une durée longue fragilise les personnes, et les rend vulnérables à la survenue de l'événement qui précipite la rupture. La 
fragilisation concerne tant le patient que son aidant, confronté à un risque d'épuisement en raison d'une sur-charge physique et mentale. Plusieurs facteurs expliquent la durée de cette période : le repérage plus ou moins précoce de la situation, la capacité des professionnels et du patient à trouver un accord sur le diagnostic de la situation et les mesures à prendre, la difficulté à trouver les ressources nécessaires, l'existence d'éventuels problèmes de coordination...

L'irréversibilité des conséquences du désajustement entre l'offre et les besoins traduit l'impossibilité de revenir à la situation préalable (GROSSETTI, 2006). Associée à la durée du désajustement, cette notion apparaît pertinente pour qualifier le niveau de risque de rupture. A titre d'exemple, l'absence prolongée de suivi médical pour certains patients crée les conditions d'une aggravation de leur pathologie que même la reprise ultérieure d'une prise en charge ne pourra pas compenser.

Le croisement de ces paramètres permet d'identifier quatre types de situations, conduisant à détailler la typologie évoquée plus haut. Nous montrons en effet que les situations de rupture avérée se distinguent par leurs suites : certaines présentent une probabilité élevée d'être surmontées grâce à la constitution d'un équilibre certes différent de celui qui prévalait avant la rupture, mais réel et adapté à la situation du patient ; d'autres ruptures au contraire ne sont jamais complètement surmontées, au sens où la nouvelle séquence est imparfaitement équilibrée et peu satisfaisante. Le cas extrême est bien sûr celui du décès à la suite de la rupture, mais d'autres situations, telles que celle déjà évoquée de la privation durable d'un suivi médical, dégradent les fondements du nouvel équilibre. Le tableau ci-dessous présente les différents cas de figure.

Les ruptures de parcours de santé : essai de typologie

\begin{tabular}{|c|c|c|c|}
\hline \multicolumn{2}{|c|}{ Paramètres } & \multicolumn{2}{c|}{$\begin{array}{c}\text { Durée du désajustement entre l'offre } \\
\text { et les besoins }\end{array}$} \\
\cline { 3 - 4 } & \multirow{2}{*}{$\begin{array}{c}\text { Courte } \\
\text { Irréversibilité des } \\
\text { conséquences du } \\
\text { désajustement entre } \\
\text { l'offre et les besoins }\end{array}$} & $\begin{array}{c}\text { Rupture évitée (M. } \\
\text { et Mme C.) }\end{array}$ & $\begin{array}{c}\text { Situation } \\
\text { déficitaire (Mme } \\
\text { B.) }\end{array}$ \\
\cline { 3 - 4 } & \multirow{2}{*}{ Forte } & $\begin{array}{c}\text { Rupture avérée, } \\
\text { avec une } \\
\text { probabilité forte de } \\
\text { création d'un } \\
\text { nouvel équilibre }\end{array}$ & $\begin{array}{c}\text { Rupture avérée, } \\
\text { avec une } \\
\text { probabilité faible } \\
\text { de création d'un } \\
\text { nouvel équilibre } \\
\text { (Mme A.) }\end{array}$ \\
\hline
\end{tabular}

Les situations de rupture évitée (exemple de $\mathrm{M}$. et Mme $\mathrm{C}$ ) conjuguent une brève durée de désajustement, avec des conséquences peu significatives à long terme. Le désajustement est interrompu rapidement grâce à la réévaluation de la situation et à l'actualisation des aides. Le dispositif de soins et d'aides est par ailleurs suffisamment étoffé et multi-dimensionnel pour que les désajustements éventuels ne créent pas de conséquences irréversibles.

Le couplage d'une longue durée de désajustement et de conséquences réversibles ou faiblement irréversibles crée les conditions d'une situation déficitaire. Ainsi, pour Mme B., les difficultés de coordination ont conduit à la pérennisation d'un plan d'aides et de soins partiellement inadapté, mais cette situation n'a pour l'instant pas engendré de conséquences irréversibles : la patiente demeure à son domicile et conserve une certaine autonomie. 
A contrario, une durée longue de désajustement associée à des conséquences irréversibles entraîne une rupture avérée, sans retour à l'équilibre possible. C'est le cas de Mme A., pour qui l'absence de suivi médical a sans doute précipité le décès. Dans d'autres situations, le désajustement produit des conséquences durables pour la personne, mais la durée du désajustement est suffisamment courte pour que subsiste une possibilité de construction d'un nouvel équilibre.

\section{Conclusion}

Notre étude avait pour ambition de mieux comprendre la dynamique de déroulement des parcours de santé, et d'apporter un éclairage théorique sur la notion même de rupture, et les conditions organisationnelles à l'origine de sa survenue ou de son évitement.

Grâce à la mobilisation de cadres d'analyse issus de la sociologie des parcours de vie et des sciences de gestion sur la fonction de coordination, nous montrons la nécessité de se doter d'une vision diachronique du parcours, appréhendé comme une série ininterrompue d'ajustements/désajustements entre des besoins en soins et en accompagnement, et des ressources disponibles et mobilisables sur un territoire donné. Nous soulignons également que ce processus d'ajustements ne peut se construire spontanément à partir de la seule intervention des professionnels de santé ou du secteur médico-social, mais qu'il nécessite la mise en œuvre de compétences spécifiques de coordination, permettant d'appréhender en permanence l'évolution des besoins de la personne et celle des ressources disponibles, et de mobiliser ces ressources pour les ajuster aux nouvelles caractéristiques de la situation. C'est grâce à ces compétences que des désajustements durables entre besoins et offre, risquant de produire des conséquences irréversibles, peuvent être évités. La gestion du risque de rupture nécessite ainsi pour le système de santé de se doter d'un véritable savoir en organisation porté par des acteurs de la coordination.

Notre étude soulève enfin un paradoxe managérial : elle montre en effet que les actions de coordination destinées à gérer les parcours complexes des personnes âgées nécessitent de maitriser des compétences multi-dimensionnelles, ainsi que de mobiliser et de faire travailler ensemble différentes expertises, mais cette fonction si importante souffre d'un déficit de reconnaissance, aux niveaux local et institutionnel. Les bouleversements nés de l'épidémie à Covid 19, pendant laquelle des coopérations et coordinations renforcées ont émergé, contribueront-ils à créer les conditions de la mise en visibilité et de la valorisation des acteurs de coordination?

\section{Bibliographie}

ABBOTT A. (2009) : «A propos du concept de turning point », in GROSSETTI M. et al., Bifurcations, p. 187-211. La Découverte. Recherches.

BESSIN M. (2009) : «Parcours de vie et temporalités biographiques : quelques éléments de problématique ». Informations sociales, vol. $6, \mathrm{n}^{\circ} 156$, p. 12-21.

BESSIN M., BIDART C. et GROSSETTI M. (2009) : «Les bifurcations, un état de la question en sociologie ». Bifurcations, p. 23-35. Recherches. La Découverte.

BIDART C. (2006): "Crises, décisions et temporalités : autour des bifurcations biographiques ». Cahiers internationaux de sociologie, vol. $1, \mathrm{n}^{\circ} 120$, p. 29-57. 
BLOCH M.-A., HENAUT L., SARDAS J.-C. et GAND S. (2011a) : « La coordination dans le champ sanitaire et médico-social: enjeux organisationnels et dynamiques professionnelles ». Etude réalisée pour la fondation Paul Bennetot par le Centre de Gestion Scientifique de Mines Paris Tech.

BLOCH M.-A., HENAUT L., GAND S. et SARDAS J.-C. (2011b) : « Les gestionnaires de cas : une nouvelle figure de tiers... ». Revue économique et sociale : bulletin de la Société d'Etudes Economiques et Sociales, n³, p.103-111.

BLOCH M.-A. et HENAUT L. (2014) : Coordination et parcours. La dynamique du monde sanitaire, social et médico-social. Edition Dunod.

BOUQUET B. et DUBECHOT P. (2017) : «Parcours, bifurcations, ruptures, éléments de compréhension de la mobilisation actuelle de ces concepts ». Vie sociale, vol. 2, n 18 , p. 1323.

BUTHION V. et GODE C. (2014) : «Les proches-aidants, quels rôles dans la coordination du parcours des personnes malades ? », Journal de gestion et d'économie médicales, vol. 32, n7, p. 501-519.

CARTIER T., NAIDITCH M. et LOMBRAIL P. (2014) : «Hospitalisations potentiellement évitables : une responsabilité des seuls soins de premier recours ? », Revue d'Epidémiologie et de Santé Publique, vol. 62, n4, p. 225-236.

CITTEE J. et al. (2015) : «Trajectoires de soins des patients ayant une bronchopneumopathie chronique obstructive : points de vue des acteurs hospitaliers ». Santé Publique, HS (S1), p. 177-187.

CONTANDRIOPOULOS A.-P., DENIS J.-L., TOUATI N. et RODRIGUEZ R. (2001) : «Intégration des soins : dimensions et mise en œuvre ». Ruptures, revue transdisciplinaire en santé, vol. $8, \mathrm{n}^{\circ} 2$, p. 38-52.

DENECKERE S. et al. (2012) : "Care pathways lead to better team work : Results of a systematic review ». Social Science \& Medicine, n 75 , p. 264-268.

FRATTINI, M. O. et NAIDITCH, M. (2015). Coordination d'appui au médecin traitant pour faciliter les parcours de ses patients. Santé Publique, 1(HS), 87-94.

GACHE K. et al. (2014) : « Main barriers to effective implementation of stroke care pathways in France : a qualitative study ». BMC Health Services Research, 14 : 95.

GAND S., HENAUT L. et SARDAS J.-C. (2014) : Aider les proches aidants. Comprendre les besoins et organiser les services sur les territoires. Mines ParisTech. Economie et gestion. Presse des Mines.

GANNE C. (2017) : «L'analyse séquentielle des parcours : pour une approche longitudinale et non causale des effets des interventions sociales ». Vie sociale, vol. 2, n ${ }^{\circ} 18$, p. 25-44.

GROSSETTI M. (2006): «L'imprévisibilité dans les parcours sociaux». Cahiers internationaux de sociologie, vol. $1, \mathrm{n}^{\circ} 120$, p. 5-28.

HENAUT L. et SARDAS J.-C. (2012) : Parcours de vie et aide à l'autonomie. Deuxièmes rencontres scientifiques de la CNSA.

JAEGER M. (2017) : «Une nouvelle temporalité dans l'action sociale et médico-sociale ». Vie sociale, vol. $2, \mathrm{n}^{\circ} 18$, p. $45-56$. 
KINSMAN et al. (2010) : "What is a clinical pathway ? Development of a definition to inform the debate $»$. BMC Medicine, $8: 31$.

LANGLEY A. and al. (2013) : "Process studies of change in organization and management : unveiling temporality, activity and flow". Academy of Management Journal, vol. 56, $\mathrm{n}^{\circ} 1$, p. $1-13$.

LAWAL et al. (2016) : "What is a clinical pathway ? Refinement of an operational definition to identify clinical pathway studies for a Cochrane systematic review ». BMC Medicine, 14 : 35 .

MATHIEU-FRITZ A. et ESTERLE L. (2013) : «Les médecins et le dossier santé informatisé communiquant: analyse d'une expérimentation du dossier médical personnel (DMP)». Réseaux, vol. 2, n¹78-179, p. 223-255.

MINVIELLE E. (2018) : Le patient et le système. En quête d'une organisation sur mesure. Approches innovantes du parcours de santé. Edition Seli Arslan.

NESTRIGUE C., COM-RUELLE L. et BRICARD D. (2019) : «Analyse séquentielle et déterminants des parcours de soins en phase post-aiguë d'un Accident Vasculaire Cérébral $(\mathrm{AVC}) \gg$. Institut de Recherche et Documentation en Economie de la Santé (IRDES), document de travail $n^{\circ} 89$.

SAINT-JEAN O. (2008) : Quelles marges d'innovations pour les systèmes de soins et d'aide aux malades âgés ? Gérontologie et société, vol. n³1, n¹26, p. 65-76.

VANHAECHT K. et al. (2012) : «Have we drawn the wrong conclusions about the value of care pathways ? Is a Cochrane review appropriate ? », Evaluation and the Health Professions, $\mathrm{n}^{\circ} 35$ (1), p. 28-42.

VANHAECHT K., DE WITTE K., PANELLA M. et SERMEUS W. (2009) : «Do pathways lead to better organized care processus ? », Journal of Evaluation in Clinical Practice, vol. 15, p. 782-788. 